\title{
Comportamiento productivo de 11 variedades de lechuga (Lactuca sativa L.) en sistema hidropónico NFT recirculante (Chachapoyas - Amazonas)
}

\section{Productive behavior of 11 varieties of lettuce (Lactuca sativa L.) in recirculating NFT hydroponic system (Chachapoyas - Amazonas)}

Santos Triunfo Leiva Espinoza ${ }^{1 *}$, Alcides Román Peña ${ }^{1}$, Nuri Carito Vilca Valqui ${ }^{1}$ y Juan Carlos Neri Chávez

\section{RESUMEN}

La hidroponía se presenta como una importante alternativa a la producción tradicional de hortalizas en suelo. Así, el sistema Nutrient Film Technique (NFT) tiene probados beneficios respecto a sistemas de producción convencionales y surge como una importante alternativa al creciente problema de escasez de tierras. En ese sentido en el presente estudio se evaluó el comportamiento de 11 variedades de lechuga con probable potencial para su producción bajo el sistema NFT, para lo cual se instaló un ensayo en diseño estadístico completo alzar con cinco repeticiones por variedad y 10 plantas por repetición. Se evaluaron variables como, altura de planta, longitud de raíz, diámetro de copa, diámetro del tallo, número de hojas, peso en fresco y porcentaje de materia seca, encontrándose que todas las variedades presentaron diferencias significativas en su comportamiento productivo, siendo la variedad Curly Green la de mejor rendimiento, con promedios en peso fresco de 203,36 g. Además mostró un promedio de 42 hojas por planta. Por otra parte la variedad Bohemia obtuvo un peso de $115,45 \mathrm{~g}$ por planta aunque obtuvo un mayor porcentaje de materia seca $(26 \%)$.

Palabras claves: Horticultura, hidroponía, materia seca, invernadero.

\begin{abstract}
Hydroponics is presented as an important alternative to traditional vegetable production in soil. Thus, the Nutrient Film Technique (NFT) system has proven benefits with respect to conventional production systems and emerges as an important alternative to the growing problem of land scarcity. In the present study, the behavior of 11 lettuce varieties with probable potential for their production under the NFT system was evaluated, for which an essay in complete statistical design was installed with five repetitions per variety and 10 plants per repetition. Variables such as plant height, root length, crown diameter, stem diameter, number of leaves, fresh weight and percentage of dry matter were evaluated, finding that all the varieties presented significant differences in their productive behavior. The variety Curly Green had the best yield because it reached averages of $203.36 \mathrm{~g}$, and an average of 42 leaves per plant. In the other hand, the Bohemia variety obtained a weight of $115.45 \mathrm{~g}$ by plant although it obtained a higher percentage of dry matter $(26 \%)$.
\end{abstract}

Keywords: Horticulture, hydroponics, dry matter, greenhouse.

\footnotetext{
${ }^{1}$ Universidad Nacional Toribio Rodríguez de Mendoza de Amazonas (UNTRM-A), Instituto de Investigación para el Desarrollo Sustentable de Ceja de Selva, Calle Higos Urco N 342-350-356, Calle Universitaria N³04, Chachapoyas, Perú

"Autor de correspondencia. E-mail: santos.leiva@untrm.edu.pe
} 


\section{INTRODUCCIÓN}

Las hortalizas alcanzaron un valor de 681,2 millones de soles para el periodo enero marzo de 2016 (MINAGRI, 2016). Son un componente importante de la dieta de muchos países y se consumen en diversas presentaciones. Así, la lechuga es de las hortalizas más representativas en la mesa global.

La hidroponía es una técnica de producción agrícola en la que se cultiva sin suelo y donde los elementos nutritivos son entregados en una solución líquida (Cruz, 2016). Los cultivos hidropónicos bajo invernadero representan además una alternativa en la agricultura moderna, y pueden ser utilizados tanto en las grandes explotaciones como en las pequeñas y medianas, sin la necesidad de profundos conocimientos agronómicos (Arcos et al., 2011). Los sistemas mayormente utilizados son el sistema de riego por goteo con lana de roca y el sistema NFT "Nutrient Film Tchnique" que traducido al español significa "la técnica de la película de nutriente". Los cultivos hidropónicos más rentables son tomate, pepino, pimiento, lechuga y flores cortadas (Marulanda, 2003).

La técnica de hidroponía conocida como NFT tiene sus orígenes en Inglaterra. Durante la década de los años sesenta se desarrolló este sistema para aumentar la productividad del sector de producción hidropónica. El método sufrió modificaciones en la Universidad de la Molina en Perú, donde se propuso utilizar tubos de PVC con perforaciones donde se colocan las plantas de lechuga, en unidades de producción de 10 tubos, separados de 20 a $25 \mathrm{~cm}$. Adicionalmente, se utiliza una bomba de acero inoxidable para la inyección de la solución nutritiva a presión, la cual entra por un extremo de los tubos y se recolecta al final de estos, para retornar al tanque de almacenamiento. Este ciclo permite la reutilización de los nutrientes, para un mayor aprovechamiento del recurso favoreciendo la absorción en los sistemas radiculares (González, 2008). El sistema NFT, se considera un sistema cerrado ya que no existe pérdida o salida al exterior debido a que la solución nutritiva circula como una lámina a través de las raíces (Carrasco et al., 2007).
Hoy en día la hidroponía es el método más intensivo de producción hortícola, y surge como alternativa productiva ante la creciente demanda por alimentos y la escasez de suelos que viene originando una agricultura tradicional. La hidroponía, generalmente es de alta tecnología y de fuerte capital, y es aplicada exitosamente con fines comerciales en países desarrollados (Arcos et al., 2011). En este orden de ideas se hace necesaria la investigación orientada a mejorar los sistemas de producción hidropónica con la introducción de variedades nuevas que ya han sido probadas en otras latitudes. Es así, que en el presente ensayo se evaluó el comportamiento productivo de 11 variedades de lechuga de procedencia española, en el sistema NFT instalado en el centro de producción hidropónica del Instituto de Investigación para el Desarrollo Sustentable de Ceja de Selva (INDES-CES) de la Universidad Nacional Toribio Rodriguez de Mendoza de Amazonas (UNTRM).

\section{MATERIALES Y MÉTODOS}

El ensayo se instaló en el centro de producción hidropónica del INDES-CES de la UNTRM, ubicado en la ciudad de Chachapoyas, capital del Departamento Amazonas. Para la instalación del cultivo se siguió la metodología correspondiente y definida a continuación.

Para la obtención de las plántulas se procedió con la siembra de semillas de cada una de las variedades, en bandejas germinadoras (una semilla por celda). Dichas bandejas fueron instaladas en la mesa de almacigado, la cual se ubicó dentro del invernadero. Durante los primeros cuatro días después de almacigada la semilla, se realizaron riegos con agua $(100 \%)$, para posteriormente cambiar el riego a base de solución nutritiva IA $(2 \mathrm{ml})$ y solución nutritiva IB $(1 \mathrm{ml})$ por cada litro de agua. El almácigo se condujo por tres semanas con riegos suaves y continuos, hasta obtener plántulas con una altura promedio de $5 \mathrm{a} 7 \mathrm{~cm}$, y de dos a tres pares de hojas verdaderas y desarrolladas.

Una vez que las plántulas estuvieron listas para su trasplante se procedió a acondicionar los tubos plásti- 
cos del sistema NFT. Para esto, fueron cargados con solución nutritiva diluida de las soluciones madre IA (Macronutrientes) y IB (Micronutrientes) en proporciones de 2 y $5 \mathrm{ml}$ de solución, respectivamente. EL nivel de $\mathrm{pH}$ alcanzado durante todo el desarrollo del experimento fue de 5,9 .

El trasplante se efectuó retirando las plántulas de las camas germinadoras y colocándolas en macetas hidropónicas, sin quitar el sustrato de las raíces. A continuación, fueron colocadas en el sistema NFT para su posterior desarrollo. Para la instalación del ensayo se utilizó una siembra al azar teniendo 11 variedades con cinco repeticiones compuestas por 10 plantas cada una (Tabla 1).

Tabla 1. Variedades de lechuga sometidas al ensayo

\begin{tabular}{cc}
\hline $\mathbf{N}^{\circ}$ & Variedad \\
\hline $\mathbf{1}$ & Bohemia \\
$\mathbf{2}$ & Luana \\
$\mathbf{4}$ & Cuatro Estaciones \\
$\mathbf{5}$ & Roxy \\
$\mathbf{6}$ & Tonya \\
$\mathbf{7}$ & Malice \\
$\mathbf{8}$ & Maravilla de Invierno \\
$\mathbf{9}$ & Curly Red \\
$\mathbf{1 0}$ & Tropicana \\
$\mathbf{1 1}$ & Dueth \\
\hline
\end{tabular}

Cuando las plantas alcanzaron el momento de la cosecha, aproximadamente 35 días después del trasplante, se procedió a realizar las mediciones de las variables en estudio, tales como altura de planta, longitud de raíz, diámetro de copa, diámetro del tallo, número de hojas y peso en fresco (Figura 1). Para ello se tomaron las medidas de cinco plantas al azar por cada repetición. Además se calculó el porcentaje de materia seca, colocando las plantas evaluadas en sobres de papel después de obtener su peso en fresco en el momento de la cosecha, y se procedió a secarlas en la estufa a $80^{\circ} \mathrm{C}$ por 48 horas, midiendo luego su peso seco y calculando el porcentaje de materia seca.

Con los datos obtenidos se procedió a realizar un análisis de varianza utilizando el software estadístico Statistix versión 8.0, con el fin de establecer diferencias entre las variedades. Así, y una vez detectadas estas diferencias se realizó una prueba de comparación de medias, específicamente la prueba de diferencia mínima significativa.

\section{RESULTADOS Y DISCUSIÓN}

A continuación se presentan en la Tabla 2 los resultados de la aplicación de la prueba de varianza a las principales variables estudiadas

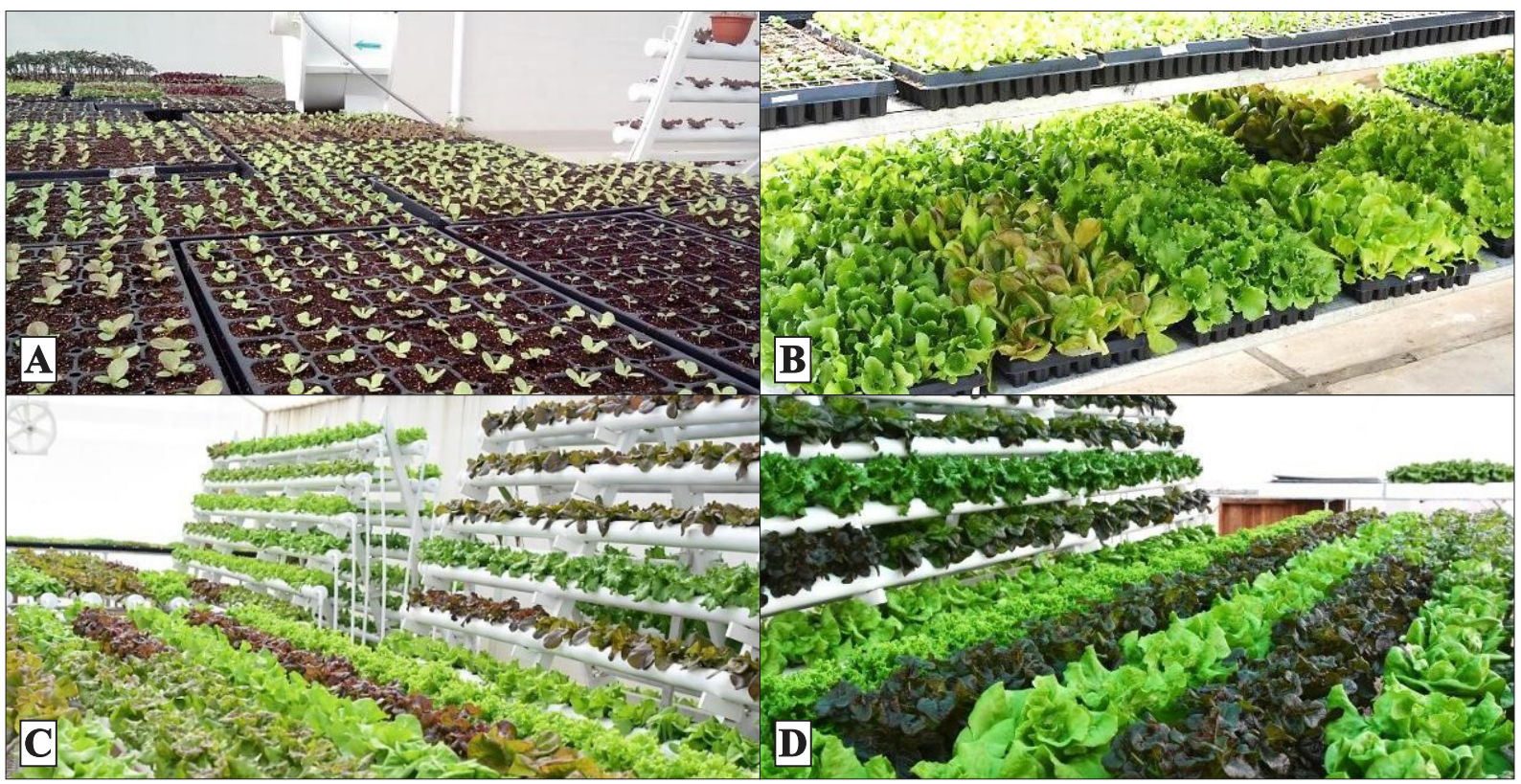

Figura 1.A: Almácigo con plántulas 8 días después de la siembra; B: Estado de plántulas listas para trasplante al sistema NFT; C: Desarrollo de las 11 variedades de lechuga, 12 días después del trasplante; D: Estado de plantas próximas a la cosecha, 25 días después del trasplante. 
Tabla 2. Análisis de varianza de las variedades instaladas

\begin{tabular}{cccc}
\hline \multirow{2}{*}{ Variable } & \multicolumn{2}{c}{$\begin{array}{c}\text { Análisis de } \\
\text { varianza }\end{array}$} & Mayor//Menor \\
\cline { 2 - 3 } & $\mathbf{F}$ & $\mathbf{P}$ & \\
\hline Altura de planta & 4,02 & $0^{* *}$ & $9 / / 5$ \\
Diámetro de copa & 7,45 & $0^{* *}$ & $3 / / 1,6,5$ \\
Diámetro del tallo & 12,01 & $0^{* *}$ & $3,11 / / 1$ \\
Longitud de raíz & 1,61 & $0.11 \mathrm{~ns}$ & IGUALES \\
Número de hojas & 48,37 & $0^{* *}$ & $5,11 / / 4,92$ \\
Peso fresco & 9,04 & $0^{* *}$ & $11 / / 8,1$ \\
Peso Seco & 18,89 & $0^{* *}$ & $9 / / 11$ \\
\% Materia Seca & 22,99 & $0^{* *}$ & $1 / / 11$ \\
\hline
\end{tabular}

**Diferencias altamente significativas

ns: Sin diferencias significativas

Los resultados de la aplicación del análisis de covarianza arrojan la existencia de diferencias altamente significativas entre los tratamientos para todas las variables, a excepción de la variable longitud de raíz en la que no se encontraron diferencias significativas entre los tratamientos.
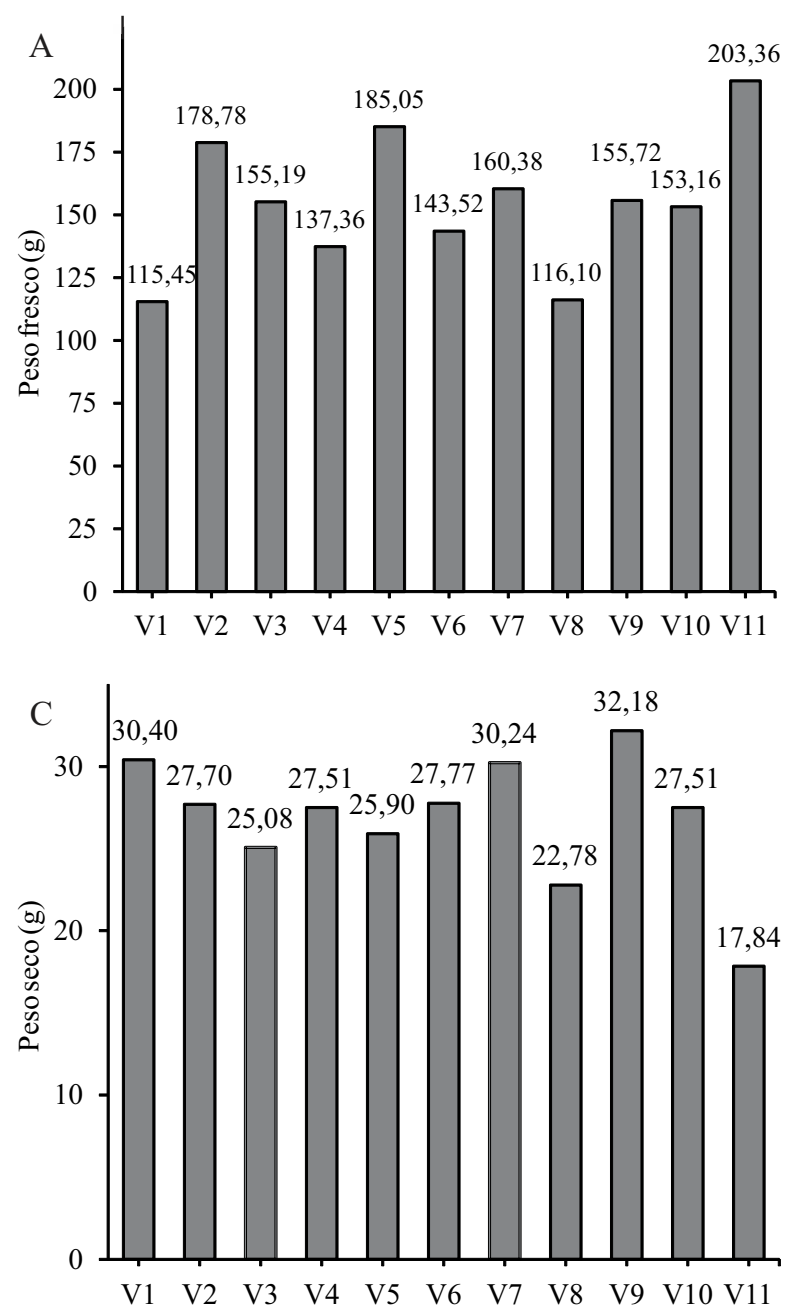

En las variables en las que se detectaron diferencias estadísticas significativas, se procedió a efectuar la prueba de comparaciones múltiples “diferencia mínima significativa", determinándose que para la altura de planta el mayor promedio lo alcanzó la variedad Tropicana (Variedad 9), mientras que para el diámetro de copa, el mayor promedio lo obtuvo la variedad Cuatro Estaciones (Variedad 3), que a su vez también alcanza el mayor diámetro de tallo. Por otro lado, el mayor número de hojas lo obtuvo la variedad Tonya (Variedad 5). Respecto a la variable peso en fresco, el mejor promedio fue alcanzado por las variedades Tonya y Luana; mientras que en contenido de materia seca el mejor promedio lo obtuvo la Variedad 1 (Bohemia).

En la Figura 2B se puede observar que el tratamiento que alcanzó la mayor altura corresponde a la variedad
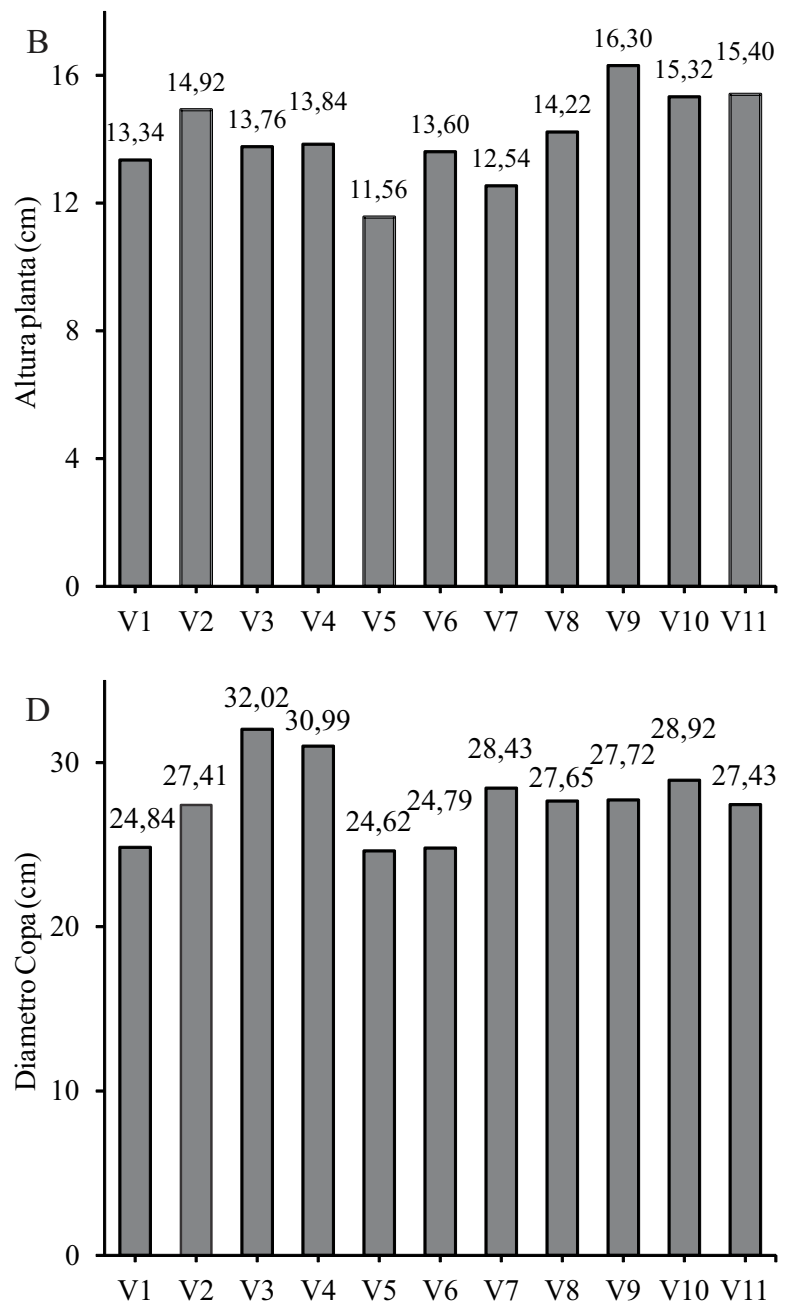

Figura 2. Principales variables estudiadas. A: Peso en fresco; B: altura de planta; C: peso en seco; D: Diámetro de copa. 
Tropicana, con 16,3 cm en promedio, mientras que la que alcanzó menor altura fue la variedad Tonya, con $11,56 \mathrm{~cm}$ de altura en promedio. Así mismo, podemos observar que, variedades como Malice, Roxy, Cuatro Estaciones y Bohemia, obtienen promedios similares, con 13,$6 ; 13,84 ; 13,76$ y $13,34 \mathrm{~cm}$ en promedio, respectivamente. Las variedades Duet y Curly Green alcanzaron promedios de altura de 15,32 y $15,4 \mathrm{~cm}$, respectivamente. Al respecto, Arcos et al. (2011), encontraron resultados similares en un ensayo trabajado con sustrato de ladrillo molido y granzón de arena, en el cual determinaron que plantas más altas con valores de 18,97 cm y 17,94 cm, y con dosis de 3 y $6 \mathrm{~g}$ de elementos mayores y elementos menores, respectivamente, se obtuvieron en el sustrato de ladrillo, mientras que el granzón de arena presentó los menores valores, de $15,47 \mathrm{~cm}$ y $15,00 \mathrm{~cm}$.

Por otro lado en la Figura 2D se muestran los valores alcanzados por el diámetro de copa por cada variedad (momento de la cosecha), siendo la variedad Cuatro Estaciones la que obtuvo el promedio más alto con $32,02 \mathrm{~cm}$., mientras que el más bajo lo presentó Tonya, con $24,62 \mathrm{~cm}$. Otra variedad con un diámetro extenso fue Roxy, con 30,99 cm de diámetro de copa en promedio.

Una tendencia similar se observa para el promedio en diámetro del tallo (Figura 3A), es la variedad Cuatro Estaciones la que obtiene el mayor promedio con $20,98 \mathrm{~mm}$, mientras que Bohemia es la que obtiene el menor promedio con 15,16 mm. Las demás variedades obtienen promedios contenidos en este rango sin muchas variaciones.

En referencia a la longitud de raíz (Figura 3D), aunque la prueba de varianza no evidencia diferencias significativas, al observar los promedios, sobresalen ciertas variaciones en dicha medida. Este es el caso de la variedad Curly Red, la cual obtuvo mayor longitud de raíz, con 23,87 cm en promedio, seguida de cerca por la variedad Malice que obtuvo 23,54 cm en promedio. La menor longitud la obtuvo la variedad Tropicana con $20,06 \mathrm{~cm}$. Al respecto, Cruz (2016) encontró que en tres variedades en estudio (Lakes, Grand Rapids Tbr y
White Boston) se mostraron largos de raíz con valores estadísticos iguales, por lo tanto las declaró homogéneas, sin embargo, las longitudes alcanzadas fueron menores a las encontradas en el presente ensayo, pues se obtuvo 18,4 cm de longitud en la variedad Great Lakes, mientras que la variedad Grand Rapids Tbr mostró un desarrollo de $18,0 \mathrm{~cm}$, y por último la variedad White Boston $16,1 \mathrm{~cm}$.

En referencia al número de hojas (Figura 3B) se evidenció que la variedad Tonya, con un promedio de 43,08 fue la que alcanzó el mayor número de hojas. Le sigue la variedad Curly Green, con 42,8 hojas en promedio. Por el contrario, las variedades con menor número de hojas son Tropicana y Luana, ambas alcanzaron un promedio de 25,96. En referencia a esta variable, Velasco et al. (2016), obtuvieron promedios de número de hoja de 14,85 hojas como máximo, siendo estos niveles muy inferiores a lo encontrado en nuestro ensayo, pero con el hándicap de que en este último estudio se usaron microorganismos y humus líquido para mejorar el rendimiento.

Al realizar las mediciones del peso fresco (Figura 2A) de cada variedad se encontró que la variedad Curly Green fue la que obtuvo mayor valor de peso fresco (203,36 g), mientras que la variedad Tonya ocupó el segundo lugar, con un promedio en peso fresco de 185,05 g en el momento de cosecha. A continuación se situó la variedad Luana con 178,78 g, sin embargo, la diferencia es notable con la variedad Bohemia que solo obtuvo en promedio 115,45 g de peso. En referencia a esta variable, Cruz (2016) encontró que la variedad Great Lakes fue superior en cuanto al peso en verde, alcanzando 134,13 g por planta. Por el contrario, la variedad Grand Rapids Tbr alcanzó 106,87 g por planta, y finalmente, la variedad White Boston alcanzó solamente $97,85 \mathrm{~g}$ por planta. Estos resultados muestran niveles de producción inferiores a los encontrados en el presente ensayo, pues la variedad Curly Green alcanzó 203,36 g por planta, y la de menor peso alcanzó 115,45 g. Así mismo, Castillo et al. (2016) comparando el sistema hidropónico y acuapónico, encontraron que el primero fue más eficiente en cuanto a bio- 
masa verde de lechuga produciendo plantas de 123,2 gr, resultados inferiores a los obtenidos en el presente estudio.

Cabe destacar que en el presente estudio se visualiza el número de hojas por planta como la variable que más contribución tienen sobre la biomasa de la lechuga; en este contexto, son las variedades Curly Green y Tonya las que, en promedio, alcanzaron el mayor nivel de peso fresco.

En referencia a la variable materia seca (MS) (Figura 3C) se observan diferencias altamente significativas entre los valores alcanzados por la variedad Bohemia, ya que en promedio, esta alcanza el mayor porcentaje de MS con 26\%. Por otra parte, la variedad Curly Green solamente obtuvo un $9 \%$ de MS, a pesar de poseer el mayor nivel de peso fresco, lo que podría evidenciar mayor nivel de acumulación de agua y un
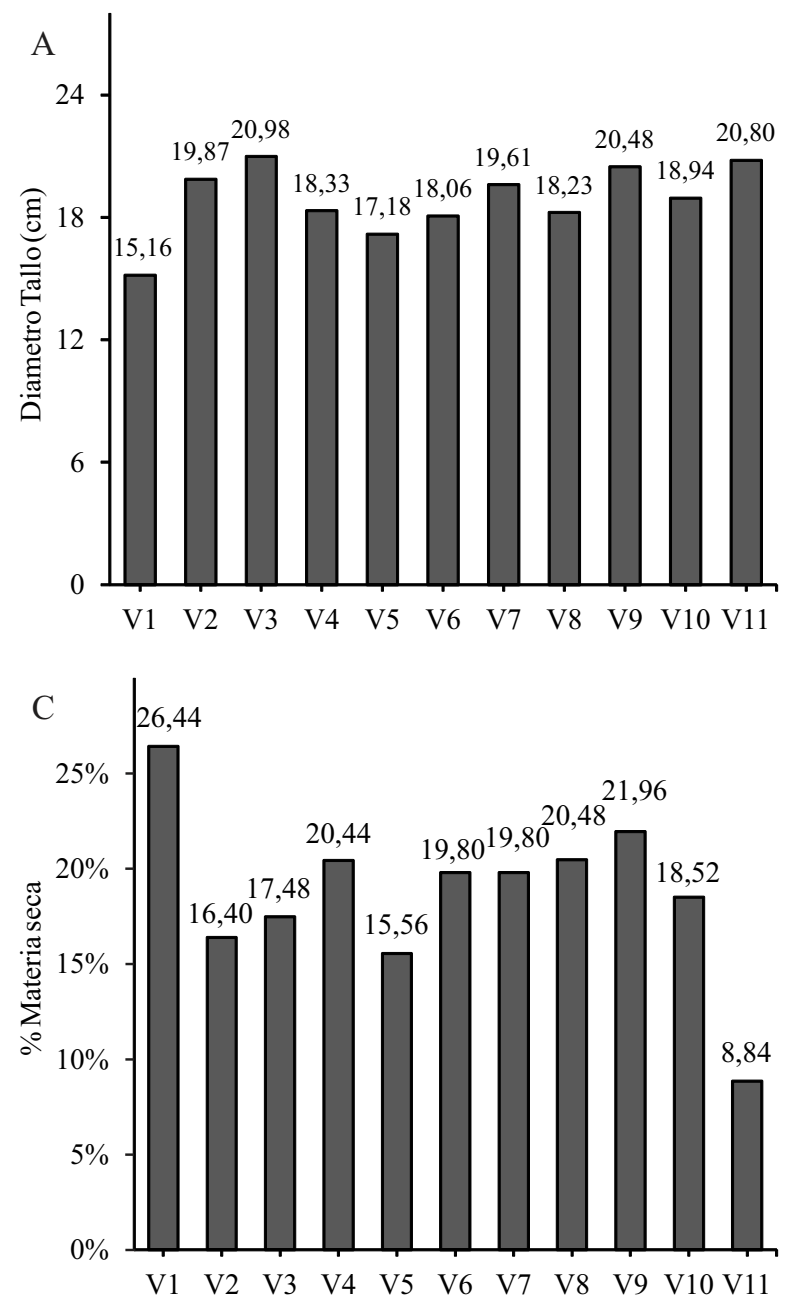

menor nivel de sólidos totales. Estos datos se ven respaldados por el cálculo de peso seco realizado en este ensayo, el cual se muestra en la Figura 2C, donde se observa que Bohemia alcanza promedios de peso seco de 30,39 g, mientras que Curly Green obtiene apenas $17 \mathrm{~g}$ de peso seco. En este sentido, Tarqui et al. (2017), utilizando la variedad Grand Rapid, obtuvieron un peso seco máximo de $10,58 \mathrm{~g}$ en lámina de riego del $100 \%$.

\section{CONCLUSIONES}

Se demuestra la existencia de diferencias altamente significativas en la respuesta al sistema Nutrient Film Tchnique NFT en las 11 variedades estudiadas, encontrándose que las variedades con mejor comportamiento respecto al peso en fresco fueron Curly Green y Tonya, mientras que la variedad con mejor comporta-
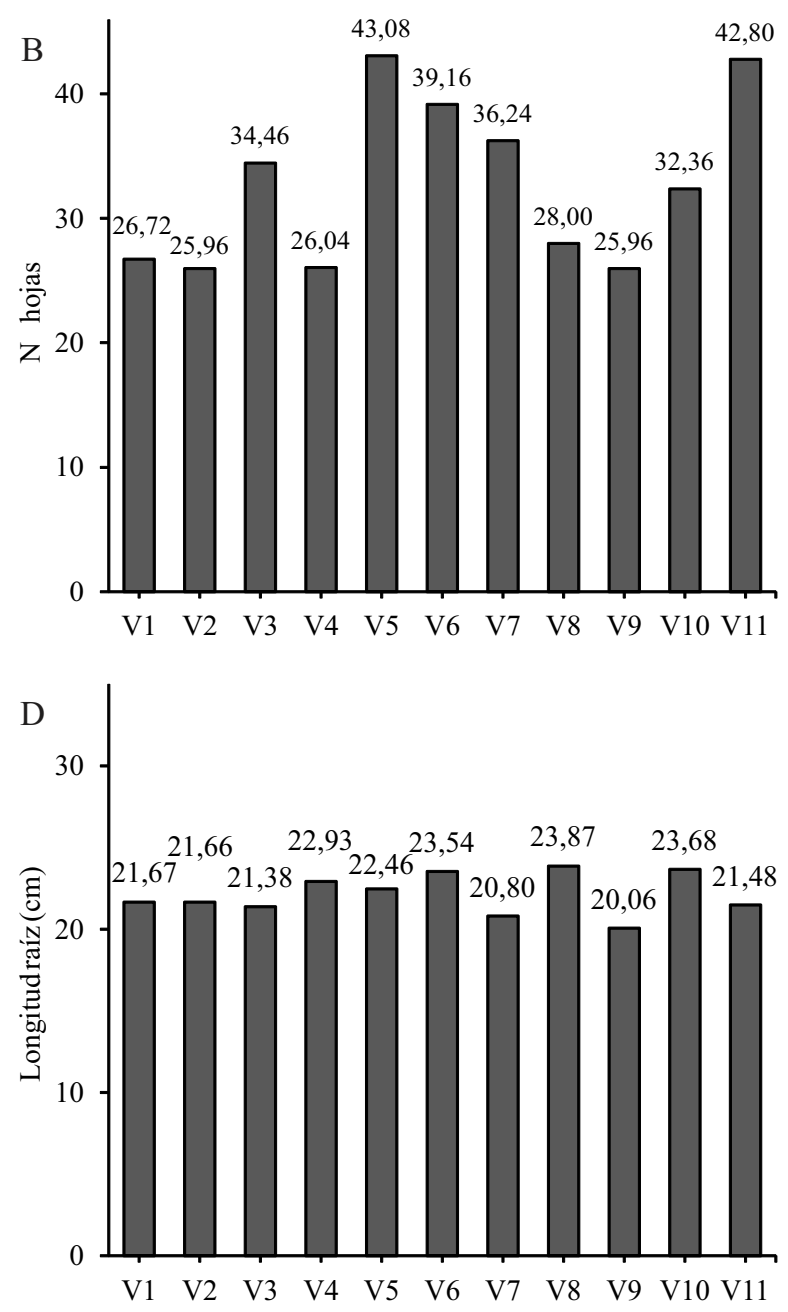

Figura 3. Detalle de variables evaluadas. A: Diámetro del tallo; B: Número de hojas; C: Porcentaje de materia seca; D: Longitud de raíz. 
miento respecto al porcentaje de materia seca fue Bohemia, con un $26 \%$.

Las variedades con mejor respuesta productiva al sistema hidropónico NFT y consecuentemente las que ofrecen un marcado potencial para producción hidropónica bajo las condiciones de agua y clima en las que se desarrolló el presente estudio, son las variedades Curly Green, Tonya y Bohemia.

Se demuestra además la efectividad del sistema NFT Nutrient Film Tchnique practicado en el INDES-CES de la UNTRM, con respecto a diferentes ensayos en otras investigaciones similares, pues se obtuvieron valores promedios superiores en las mismas variables estudiadas.

\section{REFERENCIAS BIBLIOGRÁFICAS}

Arcos, B., O. Benavides y M. Rodriguez. 2011. "Evaluación de dos sustratos y dos dosis de fertilización en condiciones hidropónicas bajo invernadero en lechuga (Lactuca Sativa L.). Revista De Ciencias Agrícolas 28 (2): 95-108.

Carrasco, G., P. Ramírez, y H. Vogel. 2007. "Efecto de la conductividad eléctrica de la solución nutritiva sobre el rendimiento y contenido de aceito esencial en albahaca cultivada en NFT". Idesia 25 (2): 59-62.

Castillo, D., I. Zavala, J. M. Ruiz, A. Radilla, J. T. Nieto, C. A. Romero y J. Gonzáles. 2016. "Implementation of an experimental nutrient film technique-type aquaponic system". Aquacult Int 24: 637-646.

Cruz, A. 2016. "Evaluación de Tres Variedades del Cultivo de Lechuga (Lactuca Sativa L.) en Dos Sistemas de Hidroponía Bajo Ambiente Semi Controlado en el Centro Experimental Chocloca". Ventana cientifica 7 (12):31-39.

González, R. 2008. "Hidroponía en NFT”. Boletín del Programa Nacional Sectorial de Producción Agrícola Bajo Ambientes Protegidos 2 (10): 7-8. Recuperado de:
http://www.mag.go.cr/bibliotecavirtual/B oletinAP2(10).pdf

Marulanda, C. 2003. Hidroponía familiar cultivo de esperanzas con rendimientos de paz. Armenia, Colombia. Tesis de Grado. Universidad la Gran Colombia. Sevilla (Colombia). Recuperado de: ht tp://www.pnud.org.co/img upload/hidroponia2004.pdf

MINAGRI - Ministerio de Agricultura y Riego. 2016. Boletín Estadístico de producción Agrícola, Pecuaria y Avícola. Lima: Sistema Integrado de Estadística Agraria. Lima(Perú): MINAGRI.

Tarqui, M., R. Chipana, F. C. Mena, J. J. Quino, R. Tallacagua y S. Gutiérrez. 2017. "Índice de estrés hídrico del cultivo de lechuga (Lactuca sativa), mediante termometría infrarroja a diferentes láminas de riego". Revista de Investigación e Innovación Agropecuaria y de Recursos Naturales 4 (1): 7-18.

Velasco, J., G. Aguirre y N. Ortuño. 2016. "Humus líquido y microorganismos para favorecer la producción de lechuga (Lactuca sativa var. Crespa) en cultivo de hidroponía". Journal of the Selva Andina Biosphere 4 (2): 71-83. 